\title{
Latest Developments on a W-band Gyro-TWA
}

\author{
W. He, C. R. Donaldson, L. Zhang, P. McElhinney, K. Ronald, \\ A. W. Cross and A. D. R. Phelps \\ Department of Physics, SUPA, \\ University of Strathclyde, Glasgow, G4 ONG, Scotland, UK \\ Email: w.he@strath.ac.uk
}

\begin{abstract}
The latest developments and experimental results a W-band gyro-TWA with a helically corrugated waveguide and a cusp electron gun are presented. The cusp gun generated a $40 \mathrm{keV}, 1.5 \mathrm{~A}$ axis-encircling electron beam. The gyro-TWA was predicted to output $5 \mathrm{~kW}$ with a $3 \mathrm{~dB}$ frequency bandwidth of 90-100 GHz.
\end{abstract}

Keywords: helically corrugated waveguide, gyrotron, gyro-TWA, W-band gyro-devices, cusp electron gun.

\section{Introduction}

The gyro-devices are high power coherent microwave sources that excel at high frequencies (up to the terahertz range). There are a number of applications for such devices including high resolution RADAR, plasma diagnostics, communications and medical imaging, and accurate material analysis based on electron paramagnetic resonance in conjunction with magnetic resonance and terahertz spectroscopy. Recently gyro-devices in the form of both a gyro-TWA [1] and a gyro-BWO [2,3], operating in the Wband, have been developed at the University of Strathclyde.

When the interaction region has a helical corrugation on the inner surface there exists an "ideal" eigenwave giving many benefits that can be exploited in novel gyro-devices and in pulse compression [4]. For the gyro-devices the eigenwave has an almost constant value of group velocity over a wide frequency band in the region of small axial wave numbers [5]. This dispersion can be designed to match the dispersion line of an electron cyclotron mode or its harmonics. This allows broadband microwave amplification to be achieved in a gyrotron travelling wave amplifier [6].

To drive the beam-wave interaction in a helically corrugated interaction region (HCIR) it is necessary to use an axis-encircling electron beam. The HCIR resonantly couples the $\mathrm{TE}_{11}$ and $\mathrm{TE}_{21}$ modes and results in a ' $\mathrm{TE}_{21}$ like' eigenmode. An axis-encircling electron beam is ideal for harmonic operation of gyro-devices as the mode selectivity nature of such a beam requires that the harmonic number is equal to the azimuthal index of a waveguide mode for effective beam wave coupling [8], which leads to a reduced possibility of parasitic oscillations. The principle of the cusp gun is based on the conservation of canonical momentum. When the electron beam passes through a magnetic field reversal an axis-encircling annular electron beam is produced [9].

\section{Cusp electron gun}

The cusp electron gun has been simulated and experimentally measured [10] to show the generation of a $1.5 \mathrm{~A}, 40 \mathrm{keV}$ axis-encircling electron beam. The alpha value has been measured over a range from 1.0 to 3.0. The results agree well with the simulations. A 3D CAD drawing of the cathode and anode can be seen in Fig. 1.

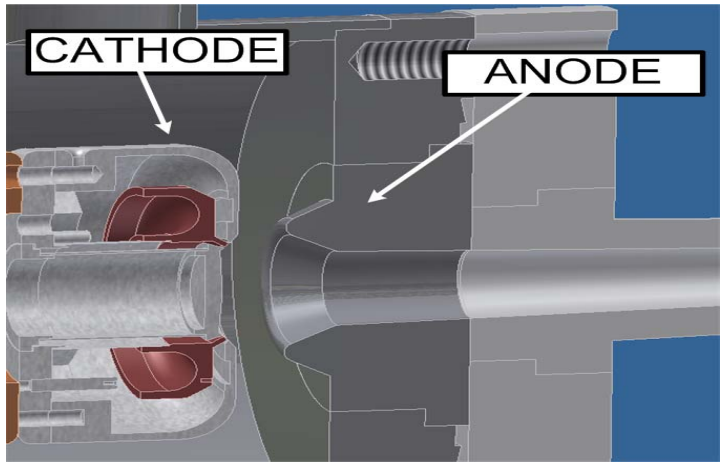

Figure 1. A CAD drawing of the cathode and anode.

The reverse coil is located behind the cathode and the main cavity coil is in front. When the magnetic field is changed between $1.65 \mathrm{~T}$ and $2.1 \mathrm{~T}$ the reverse coil's magnetic field is adjusted to keep a constant pitch angle of 1.65 . The 3D PIC code MAGIC was used to simulate and optimize the design of the cusp electron beam source.

\section{Input and output of the wave}

The input signal was inserted using an input coupler, which operated from $84 \mathrm{GHz}$ to $104 \mathrm{GHz}$. It consists of a pillbox window with a brazed ceramic disk to seal the UHV vacuum. The coupler was optimized to allow for mechanical robustness and larger machining tolerances. To allow maximum beam transportation in the coupler region, the beam tunnel incorporated a Bragg reflector at the diode side of the coupler so that the input wave would propagate downstream into the interaction region. The input coupler achieved $-10 \mathrm{~dB}$ reflection in the frequency band.

The output window, consisted of 3 disks, with the middle ceramic disk serving as the vacuum boundary and two quartz disks at both sides which achieved a low reflection of $-20 \mathrm{~dB}$ or better in the $90-100 \mathrm{GHz}$ frequency band.

Gyro-TWA beam-wave interaction 
The coupling of the beam and the eigenwave in the gyroTWA that exists inside the helically corrugated waveguide can be described by the following equation [5]:

$$
\begin{aligned}
& {\left[\left(h^{2}-2 \delta\right)\left(h+\Delta_{g}-\delta / h_{0}\right)+2 \sigma^{2} / h_{0}\right]\left[h-\left(\delta-\Delta_{H}\right) / \beta_{z 0}\right]^{2}} \\
& =C^{3}\left(h+\Delta_{g}-\delta / h_{0}\right)\left\{1+\frac{2 s}{\alpha_{0}^{2} \beta_{z 0}}\left[h-\left(\delta-\Delta_{H}\right) / \beta_{z 0}\right]\right\}
\end{aligned}
$$

The resultant dispersions of the eigenwave and beam cyclotron mode as well as the small signal growth rate can therefore be calculated.

\section{Experiment}

A photograph of the device is shown in Fig. 3. Many components have been measured including: broadband input coupler, corrugated quasi-optical mode converter, dispersion of the helical interaction region and broadband microwave window. The latest experimental results including the output powers and operating frequency bands are measured and presented.

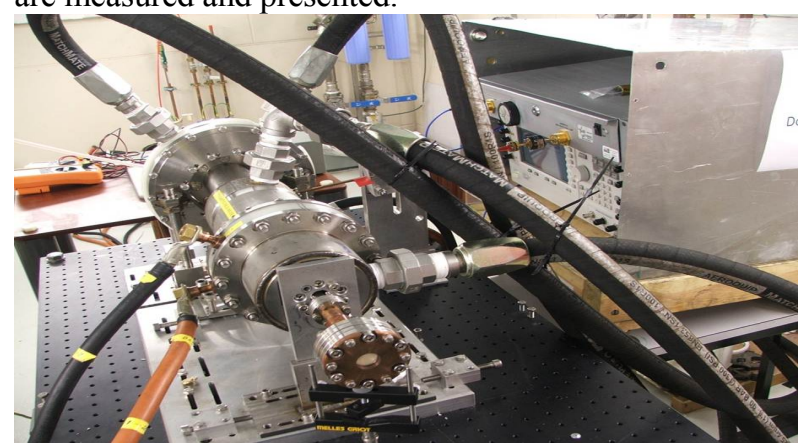

Figure 3. A photograph of the W-band gyro-device.

The output microwave radiation was detected by two crystal detectors situated inside screened boxes. The output power was calibrated using a known microwave source.

\section{Acknowledgements}

The authors would like to thank EPSRC UK (research grant EP/G036659/1) and STFC UK (research grant ST/K006673/1) for supporting this work and Dr. P. Huggard, Mr M. Beardsley and Mr. P. Hiscock of the Millimetre Wave Technology Group at the STFC Rutherford Appleton Laboratory, UK for the construction of the HCIR.

\section{References}

1. A. W. Cross, W. He, A. D. R. Phelps, K. Ronald, C. G. Whyte, A. R. Young, C. W. Robertson, E. G. Rafferty, and J. Thomson, "Helically corrugated waveguide gyrotron traveling wave amplifier using a thermionic cathode electron gun," Appl. Phys. Lett., 90, (25), 253501, 2007.
2. W. He, C.R. Donaldson, L. Zhang, K. Ronald, et al., "High power wideband gyrotron backward wave oscillator operating towards the terahertz region," Phys. Rev. Lett. 110, 165101, 2013.

3. W. He, A. W. Cross, A. D. R. Phelps, K. Ronald, A. R. Young, C. G. Whyte, E. G. Rafferty, C. W. Robertson, J. Thomson, S. V. Samsonov, V. L. Bratman, and G. G. Denisov, "Theory and simulations of a gyrotron backward wave oscillator using a helical interaction waveguide", Appl. Phys. Lett., 89, (9), 091504, 2006.

4. S. V. Samsonov, A. D. R. Phelps, V. L. Bratman, G. Burt, G. G. Denisov, A. W. Cross, K. Ronald, W. He, and H. Yin, "Compression of frequency-modulated pulses using helically corrugated waveguides and its potential for generating multigigawatt rf radiation," Phys. Rev. Lett., 92, (11), 118301, 2004.

5. L. Zhang, W. He, K. Ronald, A. D. R. Phelps, C. G. Whyte, C. W. Robertson, A. R. Young, C. R. Donaldson, and A. W. Cross, "Multi-Mode Coupling Wave Theory for Helically Corrugated Waveguide," IEEE Trans. Microwave Theory Tech., 60, (1), pp. 1-7, 2012.

6. V. L. Bratman, A. W. Cross, G. G. Denisov, W. He, A. D. R. Phelps, K. Ronald, S. V. Samsonov, C. G. Whyte, and A. R. Young, "High-gain wide-band gyrotraveling wave amplifier with a helically corrugated waveguide," Phys. Rev. Lett., 84, (12), pp. 2746-2749, 2000 .

7. W. He, K. Ronald, A. R. Young, A. W. Cross, A. D. R. Phelps, C. G. Whyte, E. G. Rafferty, J. Thomson, C. W. Robertson, D. C. Spears, S. V. Samsonov, V. L. Bratman, and G. G. Denisov, "Gyro-BWO experiments using a helical interaction waveguide," IEEE Trans. Electron. Devices, 52, (5), pp. 839-844, 2005.

8. K. R. Chu, "Theory of electron cyclotron maser interaction in a cavity at the harmonic frequencies," Phys. Fluids, 21, (12), pp. 2354-2364, 1978.

9. W. He, C. G. Whyte, E. G. Rafferty, A. W. Cross, A. D. R. Phelps, K. Ronald, A. R. Young, C. W. Robertson, D. C. Speirs, and D. H. Rowlands, "Axisencircling electron beam generation using a smooth magnetic cusp for gyrodevices," Appl. Phys. Lett., 93, (12), 121501, 2008

10. C. R. Donaldson, W. He, A. W. Cross, F. Li, A. D. R. Phelps, L. Zhang, K. Ronald, C. W. Robertson, C. G. Whyte, and A. R. Young, "A cusp electron gun for millimeter wave gyro-devices," Appl. Phys. Lett., 96, (14), 141501, 2010. 